\title{
How to: The Origin of Life or The Rise of Life and Death?
}

\author{
Vasily Alexandrovich Dementiev* \\ Vasily Alexandrovich Dementiev, Institute of Geochemistry and Analytical Chemistry, Russian Academy of Sciences, Russia
}

*Corresponding author: Vasily Alexandrovich Dementiev, Institute of Geochemistry and Analytical Chemistry, Russian Academy

of Sciences, Russia

\begin{tabular}{|c|c|}
\hline ARTICLE INFO & ABSTRACT \\
\hline Received: 幽 February 17, 2020 & Citation: Vasily Alexandrovich Dementiev. How to: The Origin of Life or The Rise of Life \\
\hline Published: 幽 February 27, 2020 & and Death?. Biomed J Sci \& Tech Res 26(1)-2020. BJSTR. MS.ID.004293. \\
\hline
\end{tabular}

\section{Opinion}

In the works devoted to the emergence of the simplest genetic code [1-4], it was found that this evolutionary process could successfully proceed in the world of peptides and nucleotides only under the following physical conditions.

a) Molecules of peptides and nucleotides are immersed in a kind of neutral condensed medium, where ATP molecules are present

b) The temperature of the medium is high enough so that the intense chaotic motion provides random encounters of peptide and nucleotide molecules with ATP molecules resulting in formation of increasingly complex polypeptides and polynucleotides and their associations with each other

c) The thermal motion in the medium is so intense that vigorous vibrational motions in long polymer molecules lead to their random fragmentation into chains of smaller linear dimensions.

A hypothetical scenario for the appearance of a prebiological genetic code in the world of peptides and nucleotides was proposed in the book [1]. The operability of this scenario was tested using molecular modeling methods [4]. In this case, condition 1 was implied, and conditions 2 and 3 were included in computer algorithms. Computer experiments revealed the following picture of successive events in the model world, left to its own devices. During collisions, amino acid molecules spontaneously combine, forming short peptides, which then expand their chains in collisions with amino acids or other peptides. Under the catalytic influence of a relatively long polypeptide, nucleotide molecules colliding with it form a polynucleotide chain of a specific structure. Thermal motion

separates a complex of two polymers, and the polypeptide chain becomes a free participant in chaotic motion. The polypeptide participating in this act is destroyed over time under the impact of particles of the medium, which leads to the completion of the building material for the polypeptides of a new random structure. However, in this world there is a chance of new instances of the aforementioned long polypeptide to occur, since the structure of the released polynucleotide carries information about the structure of the polypeptide that generated it. Also, collisions of amino acids or short peptides with this polynucleotide lead to the appearance of a complex of two polymers. One of the chains of the complex repeats the initial structure of the corresponding long polypeptide. So, a primitive genetic code arises and acts. In these experiments, not only the strength of the peptide bond was taken into account, but also the strength of the interaction between the radicals of amino acid residues [5]. This made it possible to understand why, in the process of evolution of the model world, polypeptides not only expand their chains, but also complicate the future protein texts along with the texts of the polynucleotides encoding them. From the described results it is clear that the prebiological stage of evolution, which led to the appearance and reproduction of protein structures with their enzymatic properties, could only happen in a fairly warm environment.

The chaotic thermal motion of particles of the medium and biologically important molecules creates conditions for both the addition reactions and the decay of long biopolymers into random fragments. Without such building materials of random structure, a set of protein structures with diverse properties could not have formed. Such a set is absolutely necessary for the next stage of evolution, the emergence and reproduction of living matter. Thermal 
destruction of biopolymers is their death. But such destruction is not a privilege of only biologically important molecules. Any organic molecules were destroyed and reappeared long before the appearance of peptides and nucleotides, as soon as suitable conditions appeared on Earth and in space. Consequently, Nature firstly ensured the processes of decay, death of organic matter. Then the evolution of the organic world led it to the emergence of processes of spontaneous emergence of protein structures and their genetic codes through death and the synthesis of these structures. It is important that these processes take place simultaneously and interdependently over a relatively short period of time. This is only way genetic memory of complex natural structures prone to death arises.

From theory [6] and in practice, we know that long linear polymers spontaneously break faster than short ones. And from biology it is known that long biopolymers play a crucial role in the living matter of modern organisms. However, there is no paradox. Nature has created the longest biopolymers. Then evolution stopped just because of the thermal instability of very

\section{ISSN: 2574-1241}

DOI: 10.26717/BJSTR.2020.26.004293

Vasily Alexandrovich Dementiev. Biomed J Sci \& Tech Res

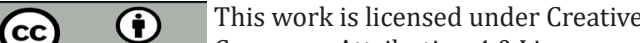
Submission Link: https://biomedres.us/submit-manuscript.php long linear biopolymers. So, we do study the rise of life and death. Or maybe we study the rise of death and life. We also believe that evolutionary processes within any complex system, up to biosphere and noosphere, take place in a series of acts of new objects birth and genetic codes creation against the necessary background of evolutionary products destruction.

\section{References}

1. EM Galimov (2001) The phenomenon of life. M: Editorial URSS.

2. VA Dementiev (2014) Driving Forces of Evolution. Geochemistry International 52(13): 1146-1189.

3. VI Baranov, LA Gribov, VA Dementiev, IV Mikhailov (2016) Some general laws of the formation of complex molecular objects in the early stages of the formation of the biosphere as a consequence of the physical properties of condensed matter. Geochemistry 11: 1046-1054.

4. VA Dementiev (2018) The emergence of a simple genetic code as a stage in the chemical evolution of the Earth. Geochemistry 1: 70-76.

5. V.A. Dementiev (2018) Interaction of radicals in polypeptides. Current Research in Biopolymers: CRBP-101.

6. LA Gribov, VA Dementiev (2010) Wave motions in molecular nanostructures: results of computer experiments. Journal of Structural Chemistry 51(2): 331-336. 